\title{
POTENCIALIDAD DE ASOCIATIVIDAD DE RESTAURANTES DEL CENTRO HISTÓRICO DE LA CIUDAD DE CARTAGENA DE INDIAS- COLOMBIA*
}

\author{
JUAN CARLOS VERGARA SCHMALBACH" ${ }^{* *}$, FRANCISCO MAZA ÁVILA \\ \& TOMÁS JOSÉ FONTALVO HERRERA \\ UNIVERSIDAD DE CARTAGENA
}

Recibido/ Received/ Recebido: 20/09/2010 - Aceptado/ Accepted / Aprovado: 22/11/2010

\begin{abstract}
Resumen
La asociatividad empresarial es una estrategia para enfrentar los mercados globalizados y la creciente competencia proveniente de otras empresas tanto del país como del exterior. El presente artículo tiene como principal finalidad medir el potencial de asociatividad para un grupo de 15 restaurantes ubicados en el centro histórico de la ciudad de Cartagena de Indias (Colombia), mediante la aplicación del método MACTOR y con el apoyo de una encuesta estructurada aplicada a potenciales asociados, siendo posible develar los objetivos comunes que se constituirán en la base de una asociación empresarial. Gracias a este método se pudo determinar un nivel de potencialidad de asociatividad del 87\%, recomendando a las empresas enfocar sus esfuerzos hacia el desarrollo conjunto de estrategias operacionales, financieras, de servicios y el fortalecimiento del talento humano.
\end{abstract}

Palabras clave: Asociaciones, asociatividad, competitividad, cooperación, método Mactor.

\section{ASSOCIATION POTENTIAL OF RESTAURANTS LOCATED AT HISTORIC CENTER OF CARTAGENA DE INDIAS CITY - COLOMBIA}

\begin{abstract}
Enterprise association is a strategy to face globalized markets and increasing competency from other national and foreign enterprises. The main objective of this article is to measure the potential of association for a group of 15 restaurants located at the historic center of Cartagena de Indias city (Colombia), through the implementation of MACTOR method and supported by a structured survey made to potential associates, in order to infer common objectives which will be the base of enterprise association. Thanks to this method, it was able to determine a level of association
\end{abstract}

Artículo científico resultado de la investigación titulada POTENCIALIDAD DE ASOCIATIVIDAD DE RESTAURANTES DEL CENTRO HISTÓRICO DE LA CIUDAD DE CARTAGENA DE INDIAS- COLOMBIA realizado por docentes del programa de Administración Industrial de la Universidad de Cartagena durante el segundo semestre de 2009 y primer semestre de 2010.

* Magister en administración, Universidad Nacional de Colombia, Investigador y Docente de Tiempo Completo Facultad de Ciencias Económicas, Universidad de Cartagena. Grupo Métodos Cuantitativos de Gestión (Categoría B). Correo electrónico: jvergaras@ unicartagena.edu.co

-.- Especialista en Gestión Gerencial, Asistente investigador Vicerrectoría de Investigación, Universidad de Cartagena. Grupo Ciencia Tecnología y Sociedad más Investigación (Categoría B). Correo electrónico: pachomaza@yahoo.com

-..' Magister en administración, Universidad Nacional de Colombia, Investigador y Docente de Tiempo Completo Facultad de Ciencias Económicas, Universidad de Cartagena. Grupo de gestión e innovación para la competitividad (Categoría B). Correo electrónico: tomasjosefontalvo@gmail.com 
potentiality of $86 \%$, recommending to enterprises to emphasize their efforts towards joint development of operational, financial, service strategies and human talent enforcement.

Key words: Associations, associative, competitiveness, cooperation, MACTOR method.

\title{
POTENCIALIDADE ASSOCIATIVA DE RESTAURANTES DO CENTRO HISTÓRICO DA CIDADE DE CARTAGENA DE ÍNDIAS (COLÔMBIA)
}

\begin{abstract}
Resumo
Aparceria de negócios é uma estratégia para competir nos mercados mundiais e afrontar o aumento da concorrência de outras empresas domésticas e estrangeiras. Neste artigo se mede o potencial de associação de um grupo de 15 restaurantes no centro histórico da cidade de Cartagena de Índias (Colômbia). Utiliza-se o método MACTOR com o apoio de um questionário estruturado aplicado a potenciais parceiros para descobrir objetivos comuns que serão a base de uma associação empresarial. Este método permitiu determinar um nível de potencialidade associativa de $87 \%$ e recomendar as empresas concentrarem seus esforços no desenvolvimento conjunto de estratégias operacionais, financeiras e de serviços, assim como no fortalecimento do talento humano.
\end{abstract}

Palavras-chave: Associações, associatividade, competitividade, cooperação, método.

Vergara, J., Maza, F. \& Fontalvo, T. (2010) Potencialidad de asociatividad de restaurantes del centro histórico de la ciudad de Cartagena de indias- Colombia. En: Revista de la Facultad de Ciencias Económicas de la Universidad Militar Nueva Granada. rev.fac.cienc.econ, XVIII (2)

JEL: D70, C02.

\section{Introducción}

Mucho se viene hablando de asociatividad, en círculos académicos, gubernamentales y hasta entre los empresarios mismos. Y no es para menos, que este concepto seduce, por su simplicidad y atractivo, como un medio de supervivencia empresarial (Liendo \& Martínez, 2001). Para nadie es un secreto que la competencia que enfrentará el empresario colombiano será cada vez más fuerte, tanto a nivel doméstico como del comercio internacional facilitado, en parte, por los tratados de libre comercio suscritos y por suscribir con algunos países y regiones; de suerte que la competencia internacional que se avecina ya no será exclusivamente contra el competidor doméstico, sino contra el competidor externo.

Ante este panorama, la asociatividad debería ser una fuente vital de auge empresarial, pero se ha convertido en un proceso bastante complejo, debido a que las empresas muy poco quieren dar a conocer información interna de sus diseños, procesos y procedimientos (Victoria, León \& Vergara, 2005). En Colombia se cuentan algunas experiencias exitosas de asociatividad empresarial (Nieto, 2006) (Riveros; Lucio Paredes; Blanco \& Harvey, 2009) (Lozano, 2010) (Gonzalo, 2010), donde la dificultad apremiante consiste en identificar aquellos intereses comunes entre el grupo de empresarios participantes.

El presente artículo propone medir el nivel de potencialidad para un grupo de restaurantes (catalogados como Pymes) representativos del sector turísticos en el centro histórico de la ciudad de Cartagena de Indias D. T. y C. (Colombia), cuyos gerentes manifestaron su deseo en participar en este estudio. Como valor agregado, se empleó el método Mactor para identificar las motivaciones, conflictos y las posibles alianzas estratégicas entre las empresas.

El sector de comercio, hoteles y restaurantes genera más de 100.000 puestos de trabajo (equivalente a 
aproximadamente el 30\% del total de empleos de la ciudad), mostrando un auge continuo con el pasar de los años (Cámara de Comercio Cartagena, 2010). Las características geográficas, sus atractivos naturales y culturales, su riqueza histórica, instalaciones hoteleras, sus vías y puertos, entre otros, convierten a Cartagena de Indias D.T. y C. en uno de los destinos principales de veraneo y negocios en el mar Caribe. En la siguiente tabla se puede observar cómo el número de pasajeros que tienen como destino la ciudad de Cartagena para fines turísticos aumentan año tras año.

Tabla 1. Listado de objetivos ${ }^{1}$

\begin{tabular}{|l|l|l|l|l|l|}
\hline & \multicolumn{1}{|c|}{$\mathbf{2 0 0 5}$} & \multicolumn{1}{|c|}{$\mathbf{2 0 0 6}$} & \multicolumn{1}{|c|}{$\mathbf{2 0 0 7}$} & $\mathbf{2 0 0 8}$ & $\mathbf{2 0 0 9}$ \\
\hline Movimiento de pasajeros de crucero & 41.542 & 42.024 & 108.892 & 206.691 & 277.507 \\
\hline Movimiento de pasajeros aéreos & 501.840 & 616.993 & 633.140 & 677.286 & 804.565 \\
\hline
\end{tabular}

En el diagnóstico situacional del sector turístico realizado por Alcaldía Mayor de Cartagena en el 2004, ya se enfatizaba la vocación turística de la ciudad como generadora de ingresos, fuentes de empleo y promotora de su desarrollo, pero a su vez, plantea varios retos de sostenimiento y mejora competitiva frente a destinos similares que cuentan con mayor inversión en infraestructura y promoción, inconvenientes que fueron manifestados por el grupo de gerentes de los restaurantes analizados.

\section{Revisión de la literatura}

La asociatividad surge como uno de los mecanismos de cooperación entre las pequeñas y medianas empresas que están enfrentando un proceso de globalización de las economías nacionales (Bernal \& La Verde, 1995), donde cada una mantiene su independencia jurídica y autonomía gerencial, decidiendo voluntariamente participar en un esfuerzo conjunto con los otros actores, para la búsqueda de un objetivo común. En general, se pueden esbozar cinco características centrales que describen la asociatividad:
- Es una estrategia colectiva basada en la cooperación de las partes.

- Se basa en una estructura flexible (generalmente en acuerdos voluntarios y no contratos que generan responsabilidad).

- No excluye a ninguna empresa por el tipo de mercado en el cual opera.

- Permite resolver problemas conjuntamente manteniendo la autonomía gerencial de las empresas participantes.

- Puede adoptar diversas formas jurídicas y organizacionales ${ }^{2}$.

Sin ser exclusiva de ellas, la asociatividad es particularmente útil para las pequeñas y medianas empresas en Colombia. La capacidad de crear asociaciones colaborativas no sólo permite la consecución de recursos, sino la posibilidad adicional de compartir modelos de gestión y procedimientos, diseño y ampliación de servicios y mecanismos de cooperación $e$ intercambio de información en tiempo real.

En Colombia, los primeros intentos por parte de la Asociación Colombiana de Pequeñas y Media-

1 Cada objetivo fue codificado mediante la letra O seguido de un consecutivo numérico. Fuente: Cartagena cómo vamos, año 2010. http://www.cartagenacomovamos.org/temas.php?iID=11

2 La Ilustración jurídica no es el primer paso para emprender un proceso asociativo. 
nas Industrias (ACOPI $)^{3}$ y otras instituciones para fomentar la asociatividad, no dieron los resultados esperados en forma inmediata; las uniones sólo buscaban resolver problemas puntuales y en el corto plazo.

"En realidad, carecían de sustancia y no atacaban el problema de fondo: crear un espíritu de grupo con una visión sobre a dónde se querían dirigir" (Pallares, 2002,11).

Diversos estudios han demostrado que para lograr que estos modelos se masifiquen de tal manera que generen un cambio en la situación socioeconómica de una región es necesario, primeramente, lograr un cambio muy importante en la cultura de las Pyme; en el caso de Cartagena:

"El pequeño y mediano empresario no está acostumbrado a cooperar con otros; la mayoría de las relaciones que concibe con sus pares son de competencia, no de cooperación" (Espinosa, 2005, 14).

La asociatividad es la respuesta más lógica y eficiente que tendrán las Pymes colombianas para sobrevivir y crecer en el largo plazo (Bustamante, 2006). A diferencia de muchas uniones empresariales, los modelos exitosos de asociatividad suelen tener en común metas de largo plazo y una visión de contribuir al mejoramiento continuo de los asociados hacia el futuro. La verdadera asociatividad no debe centrarse en la resolución de un problema específco que varios empresarios comparten.

"La idea es crear una estrategia de supervivencia a mediano y largo plazo" (Pallares, 2002, 9).

\subsection{Objetivos de la asociatividad}

Uno de los mayores atractivos de ésta Ilustración consiste en la posibilidad de fortalecer el brazo comercial de una empresa (Cerdán, 2004). Según Liendo y Martínez (2001), los requisitos más importantes para que las empresas puedan asociarse son:

- Proyecto común

- Compromiso mutuo

- Objetivos comunes

- Riesgos compartidos sin abandonar la independencia de cada uno de los participantes.

Las empresas se asocian con miras a lograr algún objetivo en común, y para concretar tal mecanismo, lo ideal consiste en buscar socios que tengan valores comunes y cuyas habilidades se complementen entre sí, de manera que todos tengan algo para contribuir-que no existan "parásitos"- y que todos tengan algo que llevarse del grupo.

En función del objetivo que persigue el grupo, pueden generarse relaciones más o menos duraderas. En el largo plazo una asociación puede concluir en la formación de una empresa con personalidad jurídica y patrimonio propio pero, en este caso, ya deja de ser un modelo asociativo. De acuerdo al Ministerio de Comercio, Industria y Turismo (2005), el proceso asociativo exige la identificación de intereses comunes de trabajo y una oportunidad de negocio que lo dinamice. De manera general, los objetivos usualmente consisten en compartir riesgos y disminuir costos. Según Ramón Rosales (1997) y Rodrigo González (2008), algunos de los propósitos por los cuales se recurre a estos modelos, como lo presenta la Tabla 1.

3 ACOPI es una organización del orden nacional que fomenta el desarrollo de las pequeñas y medianas empresas. Inicialmente la sigla ACOPI tuvo por definición: Asociación Colombiana Popular de Industriales. Posteriormente se cambió a Asociación Colombiana de Pequeñas y Medianas Industrias. Para el año 2003 se acuñó un tercer significado: Asociación Colombiana de Pequeñas y Medianas Empresas, lo cual cambia el contexto con respecto a la anterior definición y toma en cuenta además de las empresas manufactureras a las empresas de prestación de servicios. ACOPI es una entidad privada que funciona gracias a los aportes de los empresarios. 
Tabla 1. Objetivos de los modelos ${ }^{4}$

\begin{tabular}{|c|c|c|}
\hline Financieros & Organizacionales & De comercialización \\
\hline $\begin{array}{l}\text { Acceso a financiamiento, cuando las } \\
\text { garantías que se requieren no pueden } \\
\text { ser cubiertas por cada actor en forma } \\
\text { individual, pero en grupo son cubiertas } \\
\text { proporcionalmente por parte de cada } \\
\text { uno de los participantes, } \\
\text { Compras conjuntas, } \\
\text { Inversión conjunta. }\end{array}$ & $\begin{array}{l}\text { Mejora en los procesos productivos, } \\
\text { Aplicación de nuevas formas de adminis- } \\
\text { tración, } \\
\text { Implantación de planeamiento estratégico, } \\
\text { Intercambio de información productiva o } \\
\text { tecnológica, } \\
\text { Capacitación conjunta, } \\
\text { Generación de economías de escala, } \\
\text { Acceso a recursos o habilidades críticas, } \\
\text { Acceso a tecnologías de productos o pro- } \\
\text { cesos, } \\
\text { Aumento del poder de negociación, } \\
\text { Investigación y desarrollo. }\end{array}$ & $\begin{array}{l}\text { Lanzamiento de nuevos productos al mer- } \\
\text { cado, } \\
\text { Apertura de nuevos mercados, } \\
\text { Intercambio de información comercial, } \\
\text { Investigación de mercados, } \\
\text { Alianzas para vender, } \\
\text { Servicios post venta conjuntos, } \\
\text { Inversión conjunta, } \\
\text { Logística y distribución. }\end{array}$ \\
\hline
\end{tabular}

\section{Metodología}

\subsection{Método}

La investigación realizada buscó medir la viabilidad de asociación-o potencialidad de asociación- entre un grupo de empresas provenientes del mismo sector, mediante el uso de una encuesta estructurada, y empleando para su análisis el Método de Matriz de Alianzas y Conflictos: Tácticas, Objetivos y Recomendaciones -Mactor para el análisis relacional entre objetivos y empresas. Esta información preliminar permitió un diagnóstico completo, así como la creación de asociaciones basadas en objetivos comunes.

El método de análisis de juego de actores, Mactor, busca valorar las relaciones de fuerza entre los actores y estudiar sus convergencias y divergencias con respecto a un cierto número de posturas y de objetivos asociados (Godet, 2003). A partir de este análisis, el objetivo de la utilización del método Mactor consiste en facilitar a un actor una ayuda para la decisión de la puesta en marcha de su política de alianzas y de conflictos. El método Mactor comprende 7 fases:
- Fase 1 - Construir el cuadro de "estrategias de los actores". En el apartado 3.3 se encuentra el listado de actores participantes.

- Fase 2 - Identificar los retos estratégicos y los objetivos asociados. En el apartado 3.4 se encuentra el listado de objetivos analizados en este estudio.

- Fase 3 - Situar cada actor en relación con los objetivos estratégicos. Esta fase se anticipa a la elaboración de la matriz de valoración de posición por objetivos, la cual proporciona la información acerca de cómo se encuentra la empresa con respecto a los objetivos contemplados (a favor, en contra, neutral o indiferente), simplificados mediante la siguiente escala de valores:

-1: A la empresa no le interesa alcanzar el objetivo

0: A la empresa le es indiferente alcanzar el objetivo.

1: A la empresa le interesa alcanzar el objetivo

4 Los autores. 
- Fase 4 - Jerarquizar para cada actor sus prioridades de objetivos. Contempla la fase de la aplicación de la encuesta. En la Ilustración 1 del apartado 4 se puede observar la matriz de valoración de posición.

- Fase 5 - Evaluar las relaciones de fuerza de los actores. Se evalúa la cantidad de actores que están interesados por cada objetivo (esta relación se diagrama mediante un diagrama tipo balanza).

- Fase 6 - Integrar las relaciones de fuerza en el análisis de convergencias y de divergencias entre actores. Se establecen las relaciones entre actores y objetivos, analizando de forma global, los posibles enlaces asociativos.

\section{- Fase 7 - Formular las recomendaciones} estratégicas.

\subsection{Datos}

Para el estudio se recolectaron datos provenientes de quince (15) restaurantes clasificados como Pymes (de una población de 17 restaurantes identificados para un error máximo de $+/-3 \%$ ), ubicados en la zona turística de la ciudad de Cartagena de Indias (Colombia). El proceso de recolección de información primaria se desarrolló durante el primer semestre de 2010, periodo en el cual fueron contactados los gerentes de dichos establecimientos, quienes voluntariamente aceptaron ser entrevistados y respondieron una encuesta estructurada enfocada en los objetivos de asociatividad.

La aplicación del método Mactor implica un cierto número de limitaciones, principalmente concernientes a la obtención de la información necesaria (Godet, 1991): la reticencia de los actores a revelar sus proyectos estratégicos y los medios de acción externos. Existe una parte irreducible de confidencialidad; con todo, es posible proceder a contrastes y cruzamientos de información provenientes de di- versas fuentes de una manera útil. Por otra parte, el método presupone un comportamiento coherente de todos los actores en relación con sus finalidades; esto, aunque usualmente se encuentra a menudo en contradicción con la realidad, en este caso fue posible su utilización con la población objeto de estudio.

\subsection{Actores}

El grupo de actores lo conforman los 15 restaurantes considerados en el estudio, que se muestran en la Tabla 2.

Tabla 2. Establecimientos del estudio ${ }^{5}$

\begin{tabular}{|r|l|c|}
\hline 1 & Restaurante 8 -18 & $\mathrm{A} 1$ \\
\hline 2 & Restaurante Donde Olano & $\mathrm{A} 2$ \\
\hline 3 & Restaurante-Bar El Santisimo & $\mathrm{A} 3$ \\
\hline 4 & Restaurante Fusión & $\mathrm{A} 4$ \\
\hline 5 & Restaurante Juan Del Mar & $\mathrm{A} 5$ \\
\hline 6 & Restaurante La Bruschetta & $\mathrm{A} 6$ \\
\hline 7 & Restaurante y Vinoteca La Cava & $\mathrm{A} 7$ \\
\hline 8 & Restaurante La Vitrola & $\mathrm{A} 8$ \\
\hline 9 & Restaurante Palo Santo & $\mathrm{A} 9$ \\
\hline 10 & Restaurante Plaza de Armas & $\mathrm{A} 10$ \\
\hline 11 & Restaurante-Bar Porton de Santo Domingo & $\mathrm{A} 11$ \\
\hline 12 & Restaurante-Bar Quebracho & $\mathrm{A} 12$ \\
\hline 13 & Restaurante Café San Pedro & $\mathrm{A} 13$ \\
\hline 14 & Wine-Bar Restaurante Sibaris & $\mathrm{A} 14$ \\
\hline 15 & Restaurante Teriyaki Sushi-Bar & $\mathrm{A} 15$ \\
\hline
\end{tabular}

\subsection{Objetivos}

La encuesta se estructuró de acuerdo a los objetivos contemplados en el apartado 2, tal como se muestra en la Tabla 3. Los objetivos planteados anteriormente están estrechamente relacionados con los siguientes conceptos:

- FINANCIEROS: Dificultades para la consecución de financiación, tipo de financiamientos, créditos anteriores.

- COMERCIALES: Relaciones comerciales, confianza en el sector.

5 Fuente: Basado en Ramón Rosales (1997) y Rodrigo González (2008). 
- ADMINISTRATIVOS: Intercambio de información gerencial.

- OPERATIVOS: Compartir, analizar y mejorar los métodos y procedimientos actuales.

- ESTRATÉGICOS: Capacitaciones conjuntas, logística y distribución. La logística y la distribución buscan gerenciar estratégicamente la adquisición, el movimiento, el almacenamiento de productos y el control de inventarios.

- MEJORA DE SERVICIOS: Diseño y complementación de servicios. Programas de investigación y desarrollo.

- ASOCIATIVOS: Creación formal de la asociatividad entre restaurantes, alianzas de ventas, designación de responsabilidades y tareas.

Tabla 3. Listado de objetivos ${ }^{6}$

\begin{tabular}{|l|l|}
\hline \multicolumn{2}{|c|}{ TABLA DE OBJETIVOS } \\
\hline O1 & FINANCIEROS \\
\hline O2 & COMERCIALES \\
\hline O3 & ADMINISTRATIVOS \\
\hline O4 & OPERATIVOS \\
\hline O5 & ESTRATÉGICOS \\
\hline O6 & MEJORA DE SERVICIOS \\
\hline O7 & ASOCIATIVOS \\
\hline
\end{tabular}

\section{Resultados del estudio}

Como resultado de la aplicación de la encuesta, se los valores asociados a los intereses de cada actor respecto a los siete objetivos (ver Ilustración 1 ).

\begin{tabular}{|c|c|c|c|c|c|c|c|}
\hline $2 \mathrm{MAC}$ & 오 & 尺 & ○ & P & O & 8 & 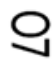 \\
\hline A1 & 1 & 1 & 1 & -1 & 1 & 1 & 1 \\
\hline A2 & -1 & 1 & 1 & 1 & 1 & 1 & 1 \\
\hline A3 & 1 & 1 & 1 & 1 & 1 & 1 & 1 \\
\hline A4 & 1 & 1 & 1 & 1 & 1 & 1 & 1 \\
\hline A5 & 0 & 0 & 1 & 1 & 1 & 1 & 1 \\
\hline $\mathrm{A} 6$ & 1 & 1 & 1 & 1 & 1 & 1 & 1 \\
\hline A7 & 0 & 1 & 1 & 1 & 1 & 1 & 1 \\
\hline A8 & 1 & 1 & 1 & 1 & 1 & 1 & 1 \\
\hline A9 & 1 & 1 & 1 & -1 & 1 & 1 & 1 \\
\hline A10 & 1 & 1 & 1 & 1 & 1 & 1 & 1 \\
\hline \begin{tabular}{|l|} 
A11 \\
\end{tabular} & 1 & 1 & 1 & 1 & 1 & 1 & 1 \\
\hline A12 & -1 & 1 & 1 & -1 & -1 & 1 & 1 \\
\hline \begin{tabular}{|l|} 
A13 \\
\end{tabular} & 0 & 1 & 0 & 1 & 1 & 1 & 1 \\
\hline A14 & 1 & 1 & 1 & 1 & 1 & 1 & 1 \\
\hline A15 & 0 & 1 & 1 & 1 & 0 & 1 & 1 \\
\hline
\end{tabular}

Ilustración 1. Matriz de valoración de posición ${ }^{7}$

Estos datos alimentaron el software MACTOR desarrollado por el ministère des Affaires étrangères de Francia ${ }^{8}$. En la Ilustración 2 se puede observar un gráfico de balanza que muestra una mayor inclinación hacia su lado positivo, deduciendo que gran parte de los restaurantes encuestados del centro histórico de la ciudad (60\%) aceptan establecer relaciones de tipo financiero con otras de las empresas encuestadas. Del lado derecho se observan las empresas que no tienen ningún tipo de interés por este objetivo (equivalente al 13\% del total de restaurantes encuestados). El resto de empresas son indiferentes a asociarse bajo un objetivo de ayuda o respaldo financiero.

El anterior resultado se debe, en gran parte, a que la mayoría de los restaurantes entrevistados manifiestan dificultades a la hora de adquirir un crédito con entidades bancarias.

\footnotetext{
6 Los autores. Cada objetivo fue codificado mediante la letra $\mathrm{O}$ seguido de un consecutivo numérico.

Fuente: elaboración de los autores. Tomado del software Lipsor-Epita-Mactor.

Se puede descargar una versión gratuita en www.3ie.org/lipsor
} 
En cuanto al análisis de los objetivos comerciales, administrativos, operativos y estratégicos, el método Mactor arrojó un alto grado de aceptación por parte de los restaurantes participantes, con una aceptación del 93\%, 93\%, 80\% y 86\% respectivamente, dando entender una apertura de procesos claves en beneficio de una mejora competitiva en la gestión de sus negocios.

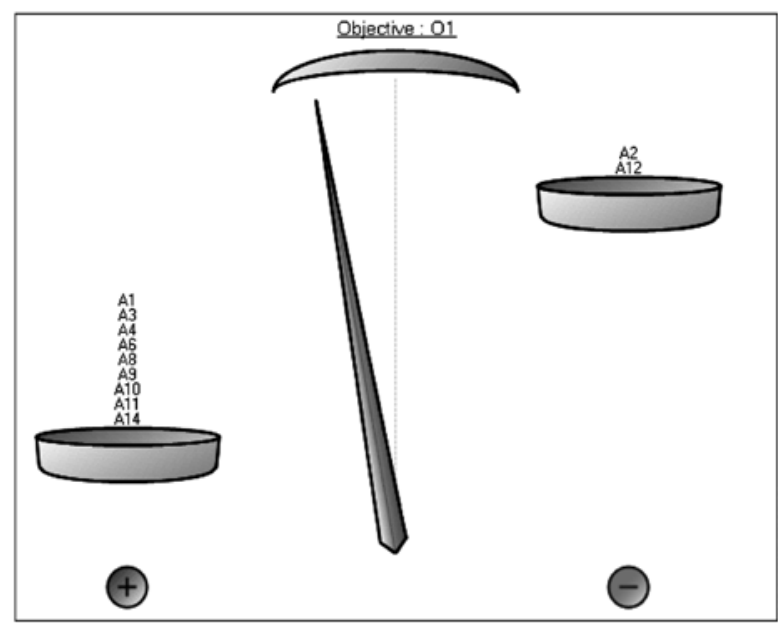

Ilustración 2. Balanza de decisión sobre el objetivo financiero $^{9}$

Por su parte, la balanza la relacionada con el objetivo de mejora de servicios tiene una inclinación total hacia su lado positivo. En consecuencia, según los resultados de las encuestas, las alianzas se orientarían a desarrollar investigaciones, desarrollos y capacitaciones en materia de mejorar la calidad del servicio ofrecido. En la Ilustración 3 se puede observar un histograma resumen sobre la implicación de los actores hacia los objetivos.

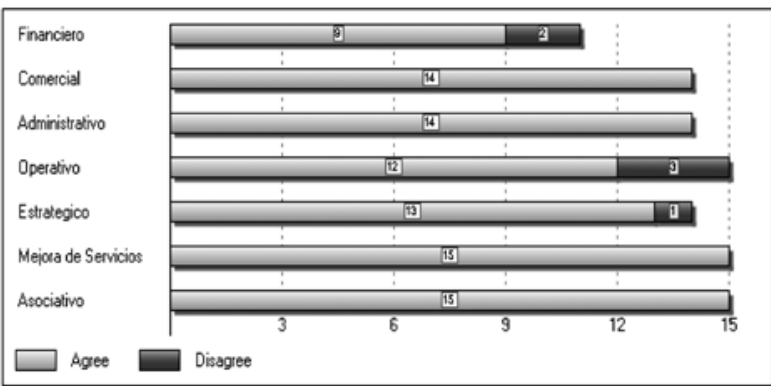

Ilustración 3. Histograma de la implicación del actor hacia sus objetivos ${ }^{10}$

El mapa de convergencia (Ilustración 4), identifica, para un par de actores, el número de posiciones comunes que tienen en los objetivos (favorables o contra). Esto identificaría el número de alianzas posibles ${ }^{11}$. Este mapa se deriva de la matriz simétrica de convergencia, y muestra las empresas que persiguen objetivos comunes. Se puede observar que A3, A4, A6, A8, A10, A11y A14 comparten muchos de los objetivos asociativos; igual sucede con A9 y A1, así como con A13 y A5.

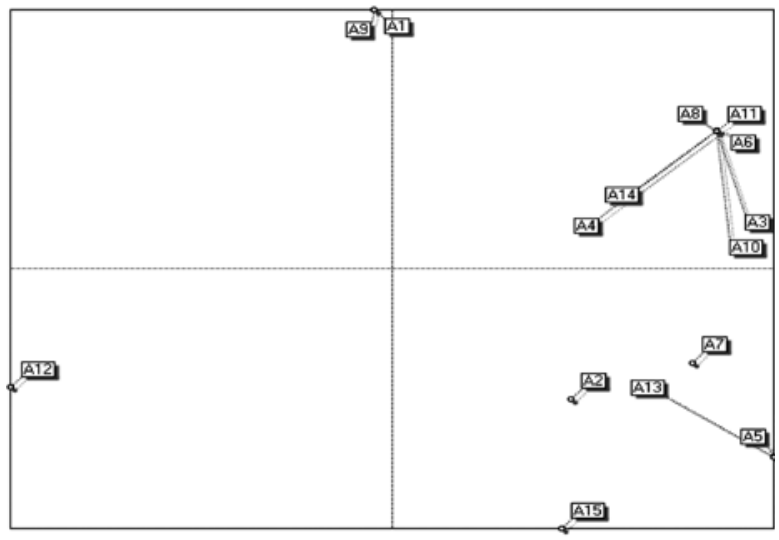

Ilustración 4. Mapa de convergencia entre actores ${ }^{12}$

\footnotetext{
9 Fuente: elaboración de los autores. Gráfico arrojado por el software Lipsor-Epita-Mactor.

10 Fuente: elaboración de los autores. Gráfico arrojado por el software Lipsor-Epita-Mactor.

11 No toman el "hilo neutro" y las posiciones "indiferentes" (cifradas como "0") en consideración. Esta es una matriz simétrica.

12 Elaboración de los autores. Gráfico arrojado por el software Lipsor-Epita-Mactor.
} 
Las otras empresas se encuentran dispersas por que sus similitudes con respecto a los objetivos son muy débiles. El gráfico de convergencia (ver Ilustración 5) identifica las relaciones entre empresas como fuertes o débiles, caracterizándolas mediante una línea conectora con un color distintivo. Los actores que están conectados por líneas rojas y azules, persiguen varios objetivos comunes, haciendo posible la creación de alianzas estratégicas entre ellas. Por su parte, el gráfico de divergencias permite ver un análisis complementario: identifica qué empresas presentan objetivos contrarios. En la llustración 6 se observa que el actor A12 presenta una divergencia muy fuerte con los actores A3, A4 A6, A8, A10, A11 y A14, al guardar posiciones diferentes en tres de los siete objetivos planteados.

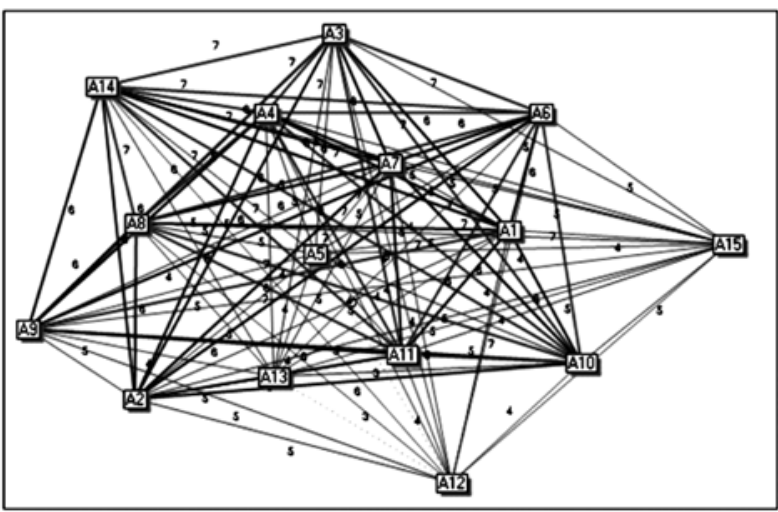

Ilustración 5. Gráfico de convergencia entre actores ${ }^{13}$

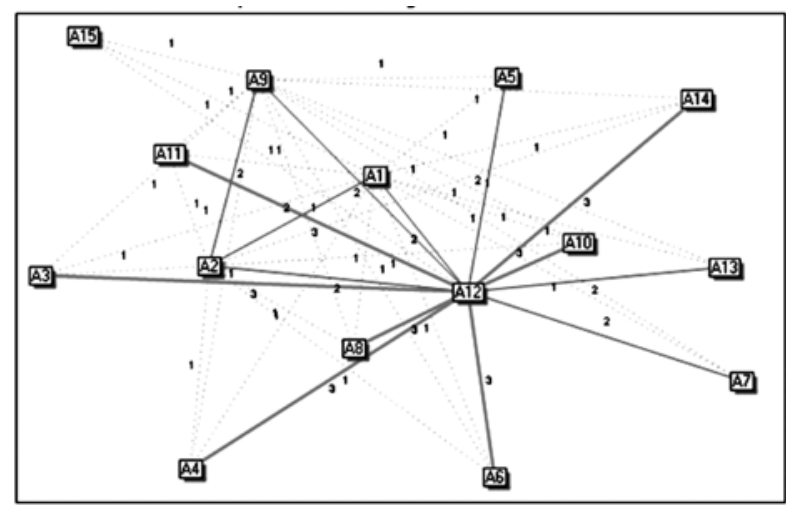

Ilustración 6. Gráfico de divergencia entre actores ${ }^{14}$

\section{Conclusiones}

La creciente participación de restaurantes en el Centro Histórico de la ciudad invita a la búsqueda de formas de asociación, para así lograr una mayor competitividad, en beneficio de sus economías y la calidad del servicio ofrecido tanto a turistas como locales. La puesta en práctica de la asociatividad entre restaurantes permitiría:

- Enfrentar y resolver problemas de manera conjunta, manteniendo la autonomía de los participantes.

- Mejorar la productividad y competitividad (mercados, negociación, costos, oportunidades, operaciones, oferta, tecnología).

- Adoptar diversas modalidades jurídicas, organizacionales y/o empresariales (sub-contratación, franquicias, alianzas).

- Aprovechar las oportunidades, neutralizar las amenazas, y poner a disposición las fortalezas de cada empresa.

- Iniciar el uso y desarrollo de la complementariedad.

El empleo de la metodología Mactor como herramienta para el análisis de objetivos entre actores para la creación de asociaciones, permite establecer los lazos fuertes y débiles a partir de los intereses comunes entre actores. En caso de crearse una asociatividad entre los restaurantes evaluados en este estudio, se debería optar por todos los objetivos según los resultados del estudio: En lo estratégico, centrados en aspectos de capacitación del talento humano y logística y distribución, áreas de gran impacto en la competitividad de estas organizaciones; en lo comercial, al mejoramiento y ampliación de la oferta de servicios turísticos y conexos. En cuanto a mejora de servicios, es sabido que la referenciación competitiva (benchmarking) es un factor que coadyuva estos procesos, los cuales se facilitan si existe la asociación, pues se evitarían los celos entre empresas; así, las firmas asociadas podrían entrar en un círculo virtuoso de mejora continua.

13 Elaboración de los autores. Gráfico arrojado por el software Lipsor-Epita-Mactor.

14 Elaboración de los autores. Gráfico arrojado por el software Lipsor-Epita-Mactor. 